\title{
Wavelength tuning of polymer optical fibre Bragg grating at longer wavelengths permanently
}

Georgios Sagias, Andreas Pospori, Daniel Gallego, David J. Webb, Horacio Lamela Rivera 


\title{
Wavelength tuning of polymer optical fibre Bragg grating at longer wavelengths permanently
}

\author{
Georgios Sagias $^{\mathrm{a}}$, Andreas Pospori ${ }^{\mathrm{b}}$, Daniel Gallego ${ }^{\mathrm{a}}$, David J. Webb ${ }^{\mathrm{b}}$, Horacio Lamela Rivera ${ }^{\mathrm{a}}$ \\ ${ }^{a}$ Opto-Electronics and Laser Technology Group (GOTL), Universidad Carlos III de Madrid, Edificio \\ Sabatini, Leganés Madrid, 28911, Spain \\ ${ }^{\mathrm{b}}$ Aston Institute of Photonic Technologies, Aston University, Aston Triangle, Birmingham, B7 4ET, \\ United Kingdom
}

\begin{abstract}
Permanent Bragg wavelength tuning of polymer optical fibre Bragg grating (POFBG) at longer wavelengths has been demonstrated for the first time utilising the thermal annealing process. In general, exposing the polymer material above its $\beta$-transition temperature, the fibre shrinks in length, the Bragg grating period becomes shorter and the Bragg wavelength shifts permanently to shorter wavelengths. In this work, a positive tuning of Bragg wavelength has been shown to be feasible when the polymer fibre is stretched during its thermal exposure. The results show that the degree of Bragg wavelength tuning strongly depends on the applying fibre strain or equivalently stress. The work presented in this paper can be used to multiplex POFBGs at any desirable wavelength.
\end{abstract}

Keywords: Polymer optical fibre, Bragg grating, Bragg wavelength, positive tuning, thermal annealing, multiplexing

\section{INTRODUCTION}

There has been a high scientific interest in polymer optical fibre (POF) sensors recently, because of their exceptional characteristics compared with the common silica optical fibre sensors [1]. The main characteristics of POFs are high fracture toughness, high flexibility in bending, biocompatibility [2] and high failure strain [3]. POF sensors are preferred to be used in stress [4], pressure [5] and acoustic wave detection applications [6], due to their lower Young's modulus compared with silica based sensors, which provides an enhanced sensitivity on POF Bragg grating (POFBG) sensors. Furthermore, there is a wide range of applications in humidity monitoring, because of the hydrophilic nature of some polymeric materials, such as the poly(methyl methacrylate) (PMMA) [7].

Exposing the POFs above their $\beta$-transition temperature, they can shrink in length, due to relaxing of the molecular stress that was introduced during the fibre drawing process. This process is called thermal annealing, and it was first used to multiplex POFBG sensors in the same piece of POF utilizing only one phase mask [8]. The thermal annealing can offer additional benefits to POFBG sensors, such as reduced hysteresis effect [9], enhanced sensitivity to humidity [10], strain [11], stress [12] and longer operational range in temperature monitoring applications [13]. The thermal annealing process is usually performed in climatic-controlled chambers [8-11,13], but recently a cost-effective and well-controlled annealing process was demonstrated, which it is used in the work presented in this paper [12]. Until now, the POF was shrinking in length during the annealing process, and consequently the Bragg wavelength was shifting at shorter wavelengths. The rate of fibre shrinkage is strongly depended on the annealing conditions, such as temperature, humidity, annealing duration, chemical composition of material, fibre drawing conditions and thermal history of POF $[10,14,15]$.

In this study, a positive tuning of Bragg wavelength is demonstrated for the first time that is feasible, when the fibre is stretched during its thermal exposure. Therefore, the rate of fibre shrinkage also depends on the degree of external force applying on the POF during the thermal annealing process. To demonstrate the concept, the fibre was first stretched by hand, it was placed on a hot-plate for 2 seconds, and then it was removed while being stretched. A permanent positive shifting of the Bragg wavelength was introduced due to this type of annealing process. To confirm the validity of the results, a new experiment was performed later in order to control the annealing conditions and make sure to strain the fibre in its elastic limit. The POFBG was attached between a fix-support and a translation-stage, and 1\% fibre strain was applied. Then, the POFBG was annealed and the Bragg wavelength positive shifted $2.8 \mathrm{~nm}$ permanently. The same POFBG was

Third International Conference on Applications of Optics and Photonics, edited by Manuel F. M. Costa, Proc. of SPIE Vol. 10453, 1045333 · @ 2017 SPIE · CCC code: 0277-786X/17/\$18 · doi: 10.1117/12.2276375 
annealed again with $2 \%$ strain and the Bragg wavelength positive shifted additional $9.7 \mathrm{~nm}$, which means $12.5 \mathrm{~nm}$ positive shifting in total. The work presented in this paper can be used to multiplex POFBG sensors at any desirable wavelength utilising only one phase mask. The combination of thermal annealing and fibre stretching can be also used in cases where the fibre shrinkage is not desirable, and the Bragg wavelength is preferred to be kept at its original spectral position. This is usually preferred in cases where the thermal treatment is used to optimise the performance of POFBG sensors (i.e. enhanced sensitivity).

\section{FABRICATION OF POFBGS}

The POFBGs were photo-inscribed in a single-mode PMMA-based micro-structured POF using a $325 \mathrm{~nm} \mathrm{He}-\mathrm{Cd}$ laser (Kimmon IK3301R-G) and a phase mask with period of $558 \mathrm{~nm}$. Their Bragg wavelength is approximately centered at $829 \mathrm{~nm}$, considering that the refractive index of PMMA is 1.49. After the fabrication, the POFBGs were placed into FC/PC connectors. The POFBGs were monitored in reflection by utilizing a super-luminescent diode (Superlum SLD-371), an optical spectrum analyzer (HP 86142A), and a 50:50 single-mode coupler, as shown in Figure 1.

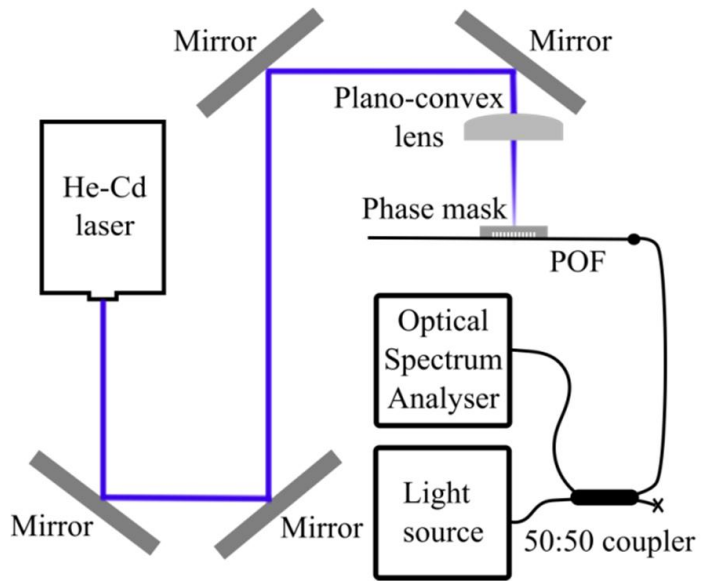

Figure 1: Setup of POFBGs fabrication

\section{EXPERIMENTAL DETAILS AND RESULTS}

Initially, to test our concept, a POFBG was stretched by hand and it was placed on a hot-plate that was heated at $75{ }^{\circ} \mathrm{C}$. After of 2 seconds, the POF was removed from the hot-plate while being stretched. At the beginning, the fibre pulling-force was not large enough and as a result the Bragg wavelength tuned from $829 \mathrm{~nm}$ to $826 \mathrm{~nm}$. The POF was placed again on the hot-plate, but with larger pulling-force this time. In this case, a permanent positive shifting of the Bragg wavelength was observed. The process was repeated 5 times and the Bragg wavelength was tuned permanently from $826 \mathrm{~nm}$ to $856 \mathrm{~nm}$. Thereafter, the POFBG was placed again on the hot-plate without any stretching in order to shift the Bragg wavelength from $856 \mathrm{~nm}$ back to $850 \mathrm{~nm}$, which was the targeted wavelength. The reflected spectrum of the POFBG before and after the annealing process is shown in Figure 2.

To confirm the validity of the results, a new experiment was performed in order to control the annealing conditions and ensure that the fibre strain is in its elastic limit. The POF was attached between a fix-support and a translation-stage (Thorlabs HFF01) and 1\% strain was applied. Then, the POF was placed in a container filled with tap water, while being strained. The water ensures that the humidity during annealing will be constant ( $100 \%$ humidity in this case). The POF remained in water for 40 minutes at ambient room temperature $\left(23 \pm 2{ }^{\circ} \mathrm{C}\right)$ in order to fully absorb the water, before the annealing process starts. After the water absorption, the Bragg wavelength stopped being shifted and the annealing process was started. The water container with the POF was placed on the hot-plate, which was turned on and we used a mercury thermometer (Fisons Thl-290-050J) to monitor the temperature (see Figure 3). The Bragg wavelength and the temperature 
were monitoring during the annealing process, as shown in Figure 4. The Bragg grating spectral position was obtained from the optical spectrum analyser. After of 45 minutes, the water was removed from the container instantly to rapidly cool down the POF. Then, the POF was placed at room ambient conditions for more than one hour, in order to release any absorbed water and the Bragg wavelength to become stabilised. The Figure 5 depicts how the Bragg wavelength positive shifted from $828.7 \mathrm{~nm}$ to $831.5 \mathrm{~nm}$.

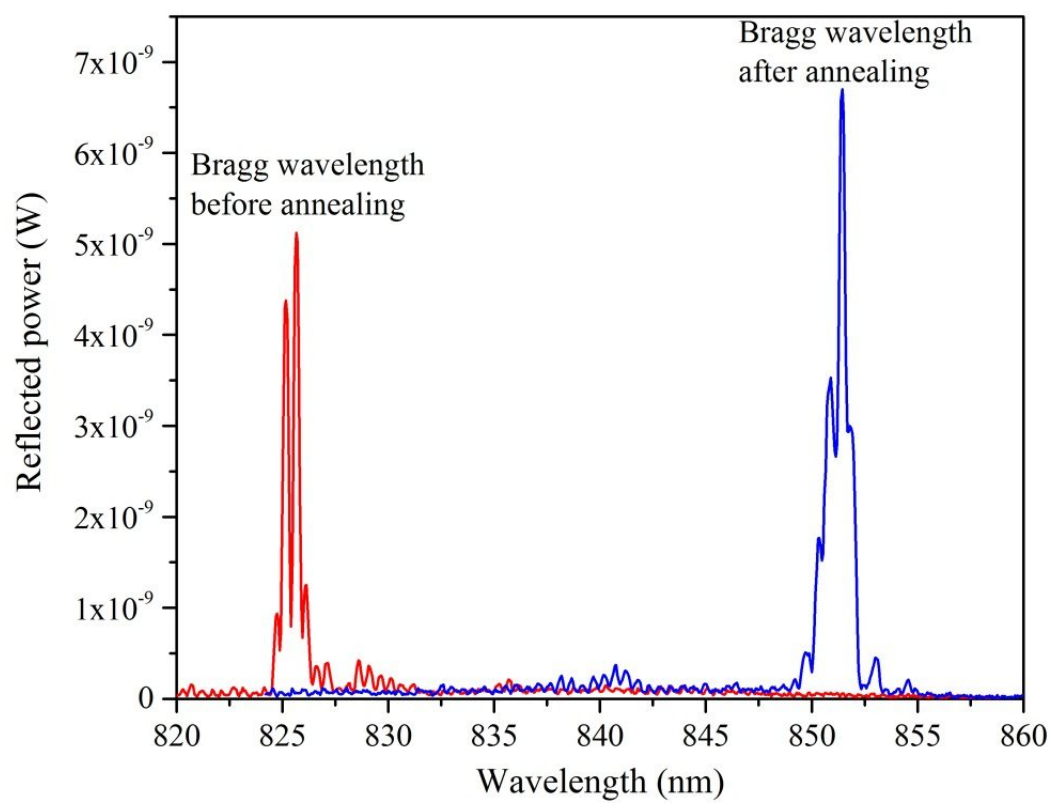

Figure 2: Bragg wavelength before and after the annealing process.

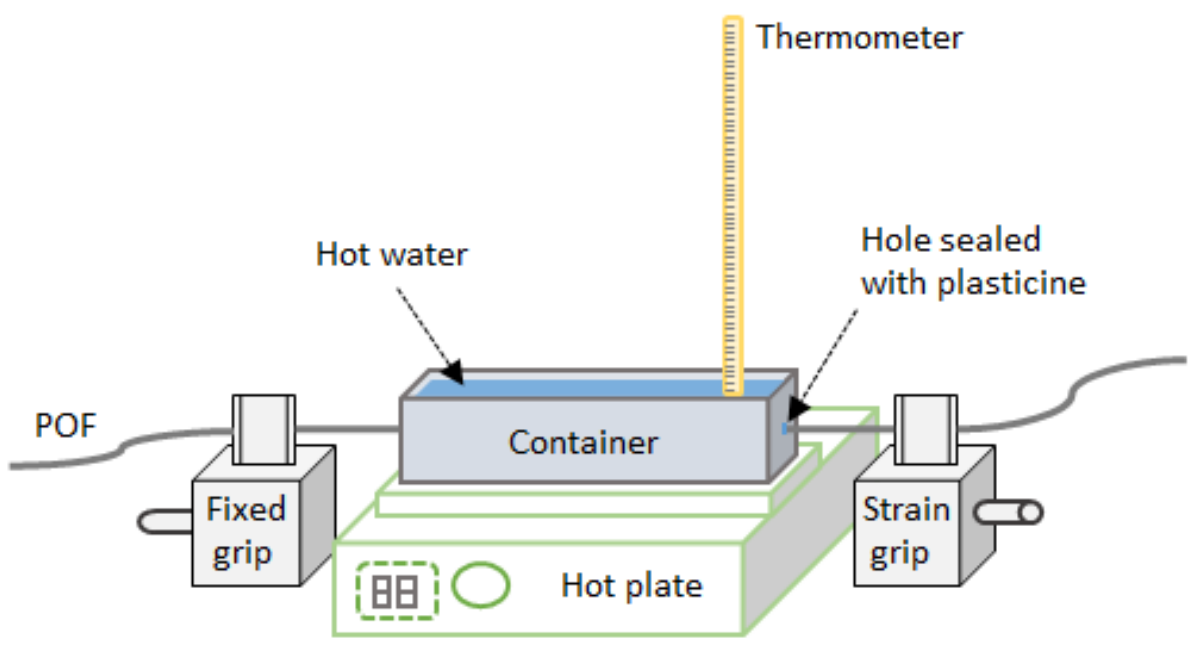

Figure 3: Experimental setup to strain and anneal the fibre. 


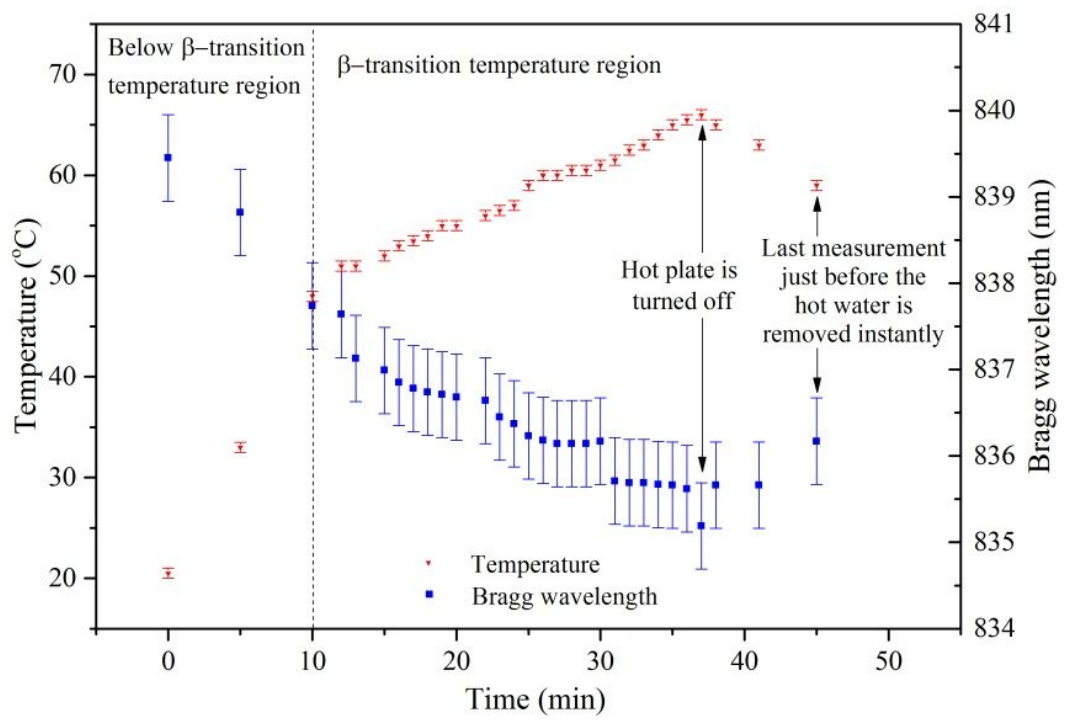

Figure 4: Bragg wavelength shift during thermal exposure and $1 \%$ fibre strain.

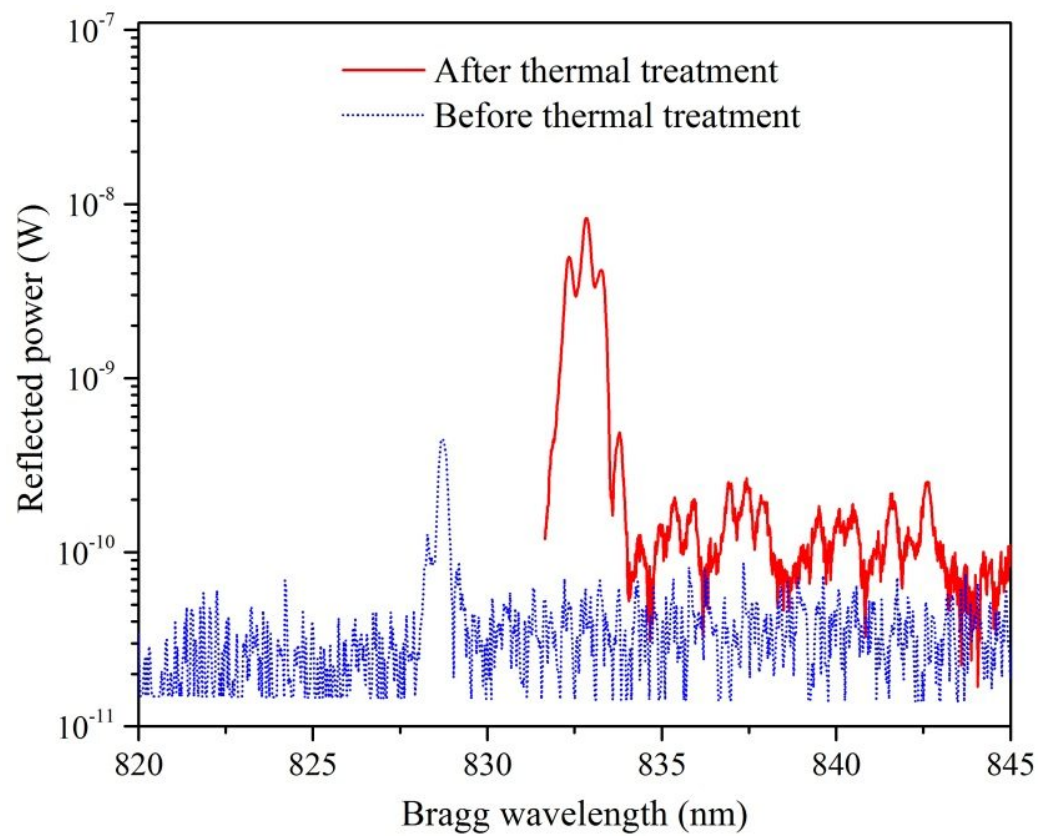

Figure 5: Bragg wavelength tuning before and after the thermal annealing treatment.

After the fibre annealing with $1 \%$ strain, the same experiment was repeated, but this time the POF was strained $2 \%$, in order to be stretched at a higher degree. The same experimental procedure was followed as before. The Bragg wavelength shift during the annealing process is shown in Figure 6. Note that the Bragg wavelength shifts at shorter wavelengths during the fibre annealing; however, if the fibre is still stretched at the end of the annealing process, releasing the applied 


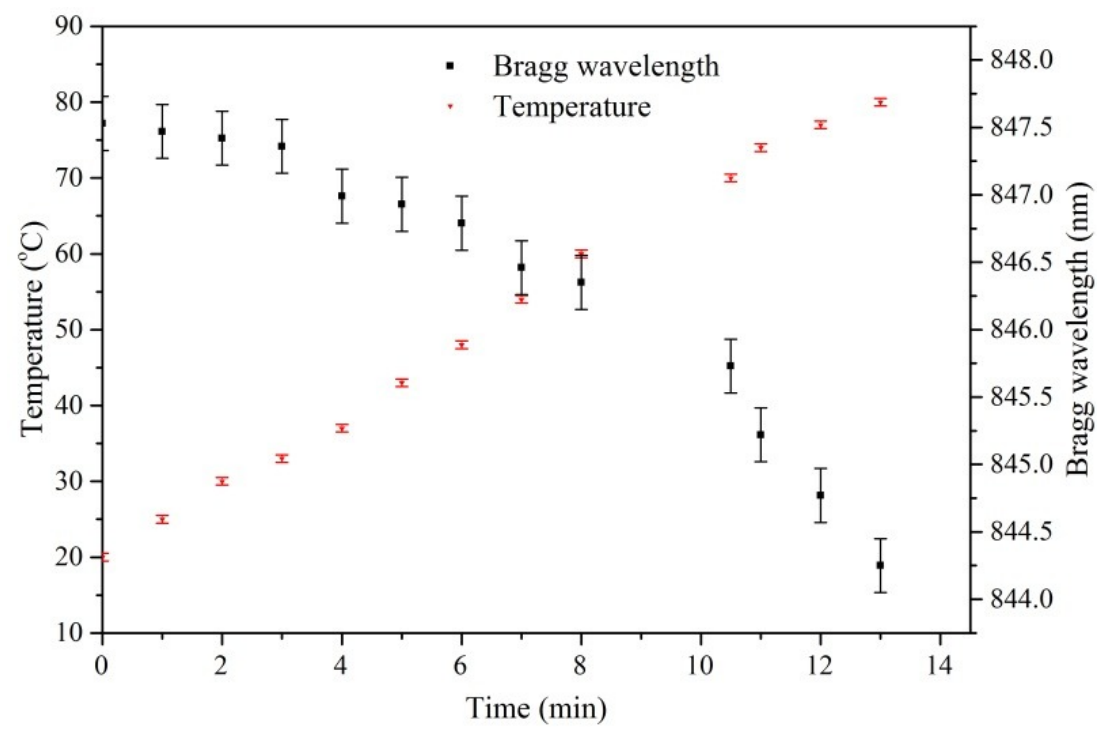

Figure 6: Bragg wavelength shift during the annealing process with $2 \%$ fibre strain.

stress, the fibre length does not return to its original length. This is the cause of the positive shifting of Bragg wavelength in POFBG devices. Annealing the POF with $2 \%$ strain, the Bragg wavelength positive shifted additional $9.7 \mathrm{~nm}$. The reason of the higher degree of tuning in this case is due to higher fibre strain (which means higher fibre stress) during the thermal annealing process. The permanent Bragg wavelength shift caused by each annealing process (with $1 \%$ and $2 \%$ strain) is illustrated in Figure 7. The results show that the Bragg wavelength was permanently shifted $12.5 \mathrm{~nm}$ to longer wavelengths.

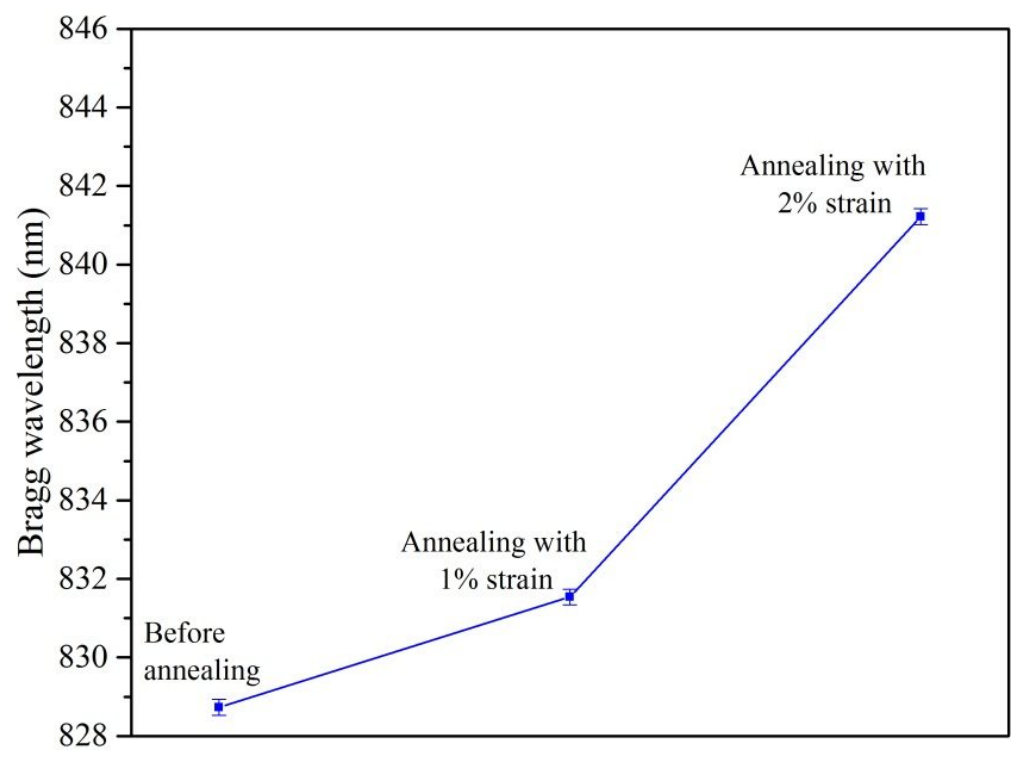

Figure 7: Bragg wavelength shift after the fibre annealing with $1 \%$ and $2 \%$. 


\section{CONCLUSION AND FUTURE WORK}

A novel POFBG multiplexing methodology is demonstrated in this work. Until now, the fibre annealing was being used to permanently tune the Bragg wavelength only to shorter wavelengths. In this paper, we show that the combination of fibre annealing and fibre stretching can be used to permanently shift the Bragg wavelength to longer wavelengths. The results show that the degree of Bragg wavelength tuning strongly depends on the applying fibre strain or equivalently stress. The work presented in this paper can be used to multiplex the POFBG sensors at any desirable wavelength utilising only one phase mask. The combination of thermal annealing and fibre stretching can be also used in cases where the Bragg wavelength needs to be kept at its original spectral position, which is feasible by appropriately selecting the degree of fibre stress during the annealing process. The POFBG sensors reported in this work will be used in future experiments to obtain optoacoustic signals for biomedical applications, evaluating their capability to be used as ultrasound detectors in a theoretical endoscope.

\section{ACKNOWLEDGEMENT}

The research leading to these results has received funding from the People Programme (Marie Curie Actions) of the European Union's Seventh Framework Programme FP7/2007-2013/ under REA grant agreement No. 608382.

\section{REFERENCES}

[1] D. J. Webb, Fibre Bragg grating sensors in polymer optical fibres, Measurement Science and Technology 26 (9) (2015) 092004.

[2] F. Bischoff, Organic polymer biocompatibility and toxicology, Clinical Chemistry 18 (9) (1972) 869-94.

[3] M. C. J. Large, J. H. Moran, L. Ye, The role of viscoelastic properties in strain testing using microstructured polymer optical fibres (mPOF), Measurement Science and Technology 20 (3) (2009) 034014.

[4] T. X. Wang, Y. H. Luo, G. D. Peng, Q. J. Zhang, High-sensitivity stress sensor based on Bragg grating in BDKdoped photosensitive polymer optical fiber, in: J. Canning, G. D. Peng (Eds.), Third Asia Pacific Optical Sensors Conference, Vol. 8351 of Proc. SPIE 8351, 2012, pp. 83510M-83510M.

[5] C. A. F. Marques, A. Pospori, D. Saez-Rodriguez, K. Nielsen, O. Bang, D. J. Webb, Aviation Fuel Gauging Sensor Utilizing Multiple Diaphragm Sensors Incorporating Polymer Optical Fiber Bragg Gratings, IEEE Sensors Journal 16 (15) (2016) 6122-6129.

[6] C. Broadway, D. Gallego, A. Pospori, M. Zubel, D. J. Webb, K. Sugden, G. Carpintero, H. Lamela, Microstructured polymer optical fibre sensors for opto-acoustic endoscopy, in: Proc. SPIE, Vol. 9886, 2016, pp. 98860S-98860S-6.

[7] W. Zhang, D. J. Webb, G. D. Peng, Investigation Into Time Response of Polymer Fiber Bragg Grating Based Humidity Sensors, Journal of Lightwave Technology 30 (8) (2012) 1090-1096.

[8] I. P. Johnson, D. J. Webb, K. Kalli, Utilisation of thermal annealing to record multiplexed FBG sensors in multimode microstructured polymer optical fibre, in: W. J. Bock, J. Albert, X. Bao (Eds.), 21st International Conference on Optical Fiber Sensors (OFS21), Vol. 7753, Proc. SPIE, 2011, pp. 7327-7753.

[9] A. Abang, D. J. Webb, Effects of annealing, pre-tension and mounting on the hysteresis of polymer strain sensors, Measurement Science and Technology 25 (1) (2014) $015102 .$.

[10] G. Woyessa, K. Nielsen, A. Stefani, C. Markos, O. Bang, Temperature insensitive hysteresis free highly sensitive polymer optical fiber Bragg grating humidity sensor, Optics Express 24 (2) (2016) 1206-13.

[11] W. Yuan, A. Stefani, M. Bache, T. Jacobsen, B. Rose, N. Herholdt-Rasmussen, F. K. Nielsen, S. Andresen, O. B. Sorensen, K. S. Hansen, O. Bang, Improved thermal and strain performance of annealed polymer optical fiber Bragg gratings, Optics Communications 284 (1) (2011) 176- 182.

[12] A. Pospori, C. A. F. Marques, D. Saez-Rodrguez, K. Nielsen, O. Bang, D. J. Webb, Thermal and chemical treatment of polymer optical fiber Bragg grating sensors for enhanced mechanical sensitivity, Optical Fiber Technology 36 (7) (2017) 68-74

[13] K. E. Carroll, C. Zhang, D. J. Webb, K. Kalli, A. Argyros, M. C. Large, Thermal response of Bragg gratings in PMMA microstructured optical fibers, Optics Express 15 (14) (2007) 8844-50.

[14] J. J. Tribone, J. M. O'Reilly, J. Greener, Analysis of enthalpy relaxation in poly(methyl methacrylate): effects of tacticity, deuteration, and thermal history, Macromolecules 19 (6) (1986) 1732-1739.

[15] P. Stajanca, O. Cetinkaya, M. Schukar, P. Mergo, D. J. Webb, K. Krebber, Molecular alignment relaxation in polymer optical fibers for sensing applications, Optical Fiber Technology 28 (2016) 11-17. 